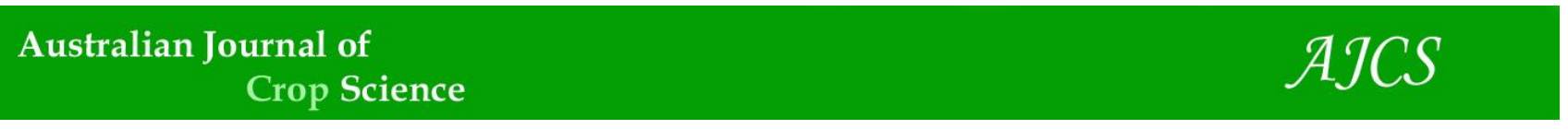

AJCS 13(09):1511-1519 (2019)

ISSN:1835-2707

doi: 10.21475/ajcs.19.13.09.p1784

\title{
Genetic inheritance of photoperiod sensitivity in Runner bean (Phaseolus coccineous L.)
}

\author{
Mable. M. Mulanya* ${ }^{1}$, Paul. M. Kimani ${ }^{1}$, Rama D. Narla ${ }^{1}$, Pascal Okwiri Ojwang ${ }^{2}$ \\ ${ }^{1}$ Department of Plant Science and Crop Protection, College of Agriculture and Veterinary Sciences, University of \\ Nairobi, Kenya \\ ${ }^{2}$ Department of Crops, Horticulture and Soils, Egerton University, Kenya
}

*Corresponding author: mulasco@gmail.com

\begin{abstract}
Lack of information on the mode of inheritance of photoperiod sensitivity is a constraint to genetic improvement of tropically adapted vegetable runner bean. This study was conducted to determine the inheritance of short-day photoperiod in runner beans. Seven single crosses between female parent; White Emergo (long day imported variety) and seven short day local landraces (Kin 1 , Kin 2, Kin 3, Nyeri, Dwarf1, Dwarf 2 and Dwarf 3) were developed in Kenya. The parents, $F_{1}, F_{2}$ and backcrosses were evaluated at Kabete (warm conditions) and OI Joro-Orok (cool condtions) under the natural short-day length of $12 \mathrm{hrs}$. From the results, short day parents flowered earlier (within 40-48 days) and formed more racemes (on average 10 racemes/plant) and pods (at least 25pods/plant) than long day parent (White Emergo) at both locations. The populations in the seven cross combinations flowered earlier at the warmer location (Kabete) than cooler one (Ol Joro Orok). In all crosses, $F_{1}$ and $F_{2}$ means of days to flowering and number of racemes were within parental range while the backcrosses' showed means that were close to their recurrent parents. The additive-dominance model $[\mathrm{m}+\mathrm{a}+\mathrm{d}]$ was found to adequately explain the genetic influence on studied traits with additive gene effects accounting for about $90 \%$ of the genetic action. The predominance of additive gene action indicates that improvement of this crop for short day adaptation can be easily achieved by trait integration through hybridization followed by pure selection methods such as single seed descent, bulk breeding or pedigree.
\end{abstract}

Keywords: Phaseolus coccineus, day length, additive and dominance effects.

Abbreviations: $\mathrm{BC}_{1} \mathrm{P}_{1}$ first backcross to female parent; $\mathrm{BC}_{1} \mathrm{P}_{2}$ first backcross to male parent; KALRO _Kenya Agricultural Livestock and Research Organization; $P_{1}$ Female parent; $P_{2}$ male parent, $F_{1}$ first filial generation and $F_{2}$ second filial generation.

\section{Introduction}

Runner bean (Phaseolus coccineus L.) is a legume species from Mesoamerica that is cultivated for dry seeds and green pods in several parts of the world. The crop is mostly cultivated in temperate countries and occasionally in highland areas of Central and South America, Africa and Asia (Brink, 2006) but at a small scale compared to common bean (Phaseolus Vulgaris L.) In Africa, runner bean is cultivated in Ethiopia, Sri-Lanka, Kenya and South Africa mainly for export. Runner bean has been traditionally grown as a grain legume crop for local use in Kenya since 2010 when commercial large scale fresh produce companies started producing vegetable runner bean for export. The traditional grain type runner bean commonly referred to 'butter bean' flowers easily under short-day conditions ( $12 \mathrm{hrs}$ ) as opposed to the vegetable runner bean varieties. The vegetable type do not flower under natural $12 \mathrm{hrs}$ day length unless there is an additional artificial light of $4 \mathrm{hrs}$. The fresh produce companies rely on imported runner bean varieties from temperate countries and it's believed that the varieties are probably adapted to long days conditions and hence do not flower when grown in conditions with a non or less promotive photoperiod (Caiger, 1995).
The vegetable runner bean is ranked by major exporters to be among the highest quality green beans in the world with Kenya being one of the leading countries in runner bean export (HCDA, 2013). Kenya has a competitive advantage to other exporters as its weather conditions favours an all year round production of this green bean. However, production of the vegetable runner in Kenya is solely done by large scale farmers because smallholder farmers are constrained by the expensive seed and installation of additional artificial light in the production fields. Therefore, breeding short-day varieties of runner beans will reduce production costs associated with additional lighting, facilitate local seed production, expand area under production and enhance smallholder production of the fresh produce. However, breeding short day grain and vegetable type runner beans is constrained by lack of information on the inheritance of photoperiod sensitivity in runner beans.

Photoperiodism influence on flowering is mainly related to the plant response to changes in relative lengths of day (Thomas and Vince-Prue, (1997) and it's a major regulator of flowering time in plants. According to Wallace et al. (1991), this sensitivity to photoperiod results into flowering vs non- 
flowering categories of genotypes. The flowering types need a particular day length to accelerate flowering even though it is not essential for flowering and in absence of such critical day length flowering is delayed. In contrast, the nonflowering need to respond to a specific day length being crucial for flowering and thus in absence of such a promotive day length the crop will not flower. Due to this variation in response to photoperiod, genotypes are classified as photoperiod-insensitive, moderately, or highly sensitive, as the quantitative delay to flowering is enlarged in response to the same non-promotive day length. Such clear categorization based on photoperiod response is unknown in runner beans and the crop is thought to be a long day or short day depending on the area of adaptation (Purse glove, 1987 and Martin, 1984).

The unclear phenomenon on inheritance of photoperiod genes in runner bean has limited improvement of this crop in the tropics where flowering of long day varieties is constrained forcing farmers to incur high production costs of installing artificial light to grow long day varieties. Up to date, information on nature of genes and gene action involved in influencing photoperiod inheritance in runner beans is scanty.

Available report on the genetic influence of photoperiod sensitivity in Phaseolus species focused on common bean. In their study, (Kornegay et al., 1993) found out that the basic photoperiod response in common bean (Phaseolus vulgaris) is controlled by two dominant genes which act in a recessive epistasis. Owing to the fact that Phaseolus coccineus has been shown to have distinct differences with Phaseolus vulgaris even though in the same genus its important to also determine the influence of photoperiod in runner bean (Phaseolus Coccineus)

We therefore studied the photoperiod gene activity in runner bean using generation means analysis. This method has been found to be the most appropriate biometrical method because it provides information on estimates of the main gene actions (additive and dominance) and epistatic effects (Ganesh and Sakila, 1999). The method was developed by Mather and Jinks (1982) and has been extensively used for both self and cross pollinated crops. It has been used to determine genetic effects of plant height in common beans (Checa et al., 2006), duration to flowering, number of pods and grain yield in lentils (Khodambashi et al., 2012; Bicer and Sarkar 2008). Despite the expansive use of generation mean analysis, the use of this method to determine gene action of photoperiod inheritance or other traits has not been reported in runner bean. Wallace et al. (1991) found that there is an interactive control of temperature on photoperiod gene activity and hence we also exposed the populations to two environments, while OlJoro Orok represented the cooler wet climates and Kabete as the warmer site for this study. Therefore, the objective of this study was to determine the inheritance of short day photoperiod in runner beans.

\section{Results}

\section{Mean performance of crosses and populations}

Analysis of variances (ANOVA) done separately for each location and cross showed significant differences $(P \leq 0.05)$ in all traits and crosses except for the number of racemes at first flowering for the cross (White Emergo x Kin 3) at both sites (Table 1). Therefore, this trait for this cross (White
Emergo $\times$ Kin 3) was excluded from further genetic analysis. From the ANOVA results, the two parents showed differences in the number of days to flowering, number of racemes and pods formed in all crosses at both locations. In all crosses, short day parents (Parent 1) flowered earlier and had more racemes and pods than long day parent (Parent 1 ). This pattern was identical with backcrosses which flowered like their respective parents. Backcrosses to White Emergo (parent 1) tended to flower late while those backcrossed to short day local landraces flowered early in all crosses at both sites (Table 1). The six populations in all crosses took longer days to flower at OlJoro-Orok. This pattern was also consistent in the number of flowers and pod formation where the backcrosses to White Emergo had fewer racemes ( 4 to 9 racemes plant ${ }^{-1}$ ) and backcrosses to short day parents having ( 8 to 14 racemes plant ${ }^{-1}$ ). The $F_{1}$ and $F_{2}$ mean of days to flowering, number of racemes and pods occurred within the range of parental means. However, $F_{1}$ generation yielded more pods than both parents in number of pods formed for the crosses involving White Emergo x Kin 1, White Emergo $x$ Dwarf 1, White Emergo x Dwarf 2 at Kabete and White Emergo $\times$ Kin 2 at OlJoro-Orok. In all crosses, the dwarf parents were found to flower early at both sites. The cross involving White Emergo x Nyeri had the highest number of racemes and pods at both sites.

The frequency distributions obtained from ANOVA results were normally distributed; a typical indication that the studied traits were quantitatively inherited. Therefore, further genetic analysis using generation mean analysis was performed to determine the gene action influencing the traits in runner beans.

\section{Genotype by environment Influence on flowering}

The relationship between mean temperature and flowering was determined by evaluating the populations at Kabete (Warm conditions) and OI Joro-Orok (Cooler conditions) but under the same day length of 12 hours. The climatic conditions during the flower induction period are presented in Table 5. Combined analysis of days to flowering showed significance differences in locations for all crosses. Significant effects of interaction between genotypes $x$ environment was observed in crosses involving White Emergo $\times$ Kin 1, White Emergo $x$ Dwarf 1 and White Emergo $x$ dwarf 2 (Table 2). In both crosses, the six populations flowered earlier at Kabete than at Ol Joro Orok including White Emergo the long day parent (Table 1).

\section{Choice of genetic model}

The $3(m+a+d)$ and $6(m+a+d+a a+a d+d d)$ parameter models were tested through regression analysis for the best fit to explain the genetic control of traits studied in the seven cross combinations. Based on the regression analysis, the 3parameter model proved to be more significant than the 6parameter model. To further ascertain the adequacy of the 3-parameter model, a joint scaling test was done. The joint scaling test results based on t-test showed that the scale tests A, B and C were not significant for all crosses and for all traits at both sites (table attached as supplementary file). This indicated the adequacy of the 3-parameter model in influencing days to flowering, number of racemes at first and second flushes and number of pods. Based on the results of the regression analysis, and the high $\mathrm{R}^{2}$ values generated for this model compared to the 6-parameter model (table 
attached as a supplementary file), the model $m+a+d$ was chosen. Since this model was found to adequately influence studied traits in the seven crosses, it was then necessary to determine the individual effect of each genetic component for each cross and trait in the chosen model [m-a-d].

\section{Estimates of gene effects based on 3 parameter model [m-} $a-d]$.

The expected mean [m] was significant and positive in traits studied and in all crosses at both sites except for first flowering trait in cross involving white Emergo x Kin 3 (Table 3). Therefore, this trait for this cross; White Emergo $\times$ Kin 3 was excluded from further genetic analysis. The analysis of the gene effects revealed that additive and dominance effects were involved in the inheritance of most traits. Additive gene effects were found positive for all traits in the seven crosses at either or both sites. Dominance effects were non-significant in most traits except for the number of pods. Both additive and dominance effects were significant in White Emergo $x$ dwarf 1 and White Emergo $x$ dwarf 2 . Based on the genetic analysis and joint scaling tests, the $(m+a+d)$ model adjusted well and showed a better fit for all crosses and traits studied at both sites (Table 3 ). The coefficient of determination $\left(R^{2}\right)$ varied in traits, crosses and locations. $R^{2}$ value ranged between 0.77-0.94 in days to flowering, $0.59-0.89$ in first flowering racemes, $0.46-0.88$ in second flowering racemes and $0.66-0.93$ in all crosses and both locations. White Emergo $x$ Nyeri cross recorded a higher R-value (0.71-0.94) in all traits.

\section{Heritability and heterosis}

Broad sense heritability was relatively high for all traits and ranged from $70-91 \%$ in days to flowering, $(71-89 \%)$ racemes at first flowering, (68-93\%) racemes at second flowering and (64-81\%) in a number of pods as shown in Table 3.13. Better parent heterosis (BPH) varied from negative to positive among crosses, traits and locations (Table 4). For days to $50 \%$ flowering, better parent heterosis was positive in the crosses; White Emergo x Kin 3 and negative in White Emergo $\mathrm{x}$ Kin 1 at both sites. However, in the crosses; White Emergo $\mathrm{x}$ Kin 2, White Emergo x Nyeri, White Emergo x Dwarf 1, Dwarf 2 and Dwarf 3 positive heterosis in days to flowering was observed at OlJoro-Orok and negative heterosis at Kabete. All crosses showed negative heterosis in the number of racemes formed at first flowering except in White Emergo $\mathrm{x}$ Kin 2 at both sites and in White Emergo x Dwarf 1 and White Emergo x Dwarf 2 at OlJoro-Orok (Table 4). For the number of racemes formed during the second flowering, positive heterosis was at least recorded in all crosses at either location apart from White Emergo x Dwarf 1 and White Emergo $\mathrm{x}$ Kin 2 at both locations. Positive heterosis was observed in all crosses and at both sites for the number of pods formed on a plant (Table 4).

Discussions

\section{Mean performance of populations and crosses}

The ANOVA results showed that $\mathrm{P}_{1}$ (female imported long day variety) and $P_{2}$ (short day male parents) were contrasting in the means of traits studied in all evaluated crosses indicating the considerable genetic diversity among the parents and their respective crosses. Also, the backcrosses $\left(B C_{1} P_{1}\right.$ and $\left.B C_{1} P_{2}\right)$ showed means that tended to be close to their respective recurrent parents. These results thus confirm the correct choice of contrasting parents in respect to day length adaptation which is a prerequisite of conducting generation mean analysis as proposed by Mather and Jinks (1971). The male parents formed more racemes, pods and flowered earlier in all crosses indicating the superior adaptation of these materials to the short-day conditions. White Emergo delayed to flower and had fewer racemes and pods revealing how flowering of this variety is constrained under short day conditions. The occurrence of $F_{1}$ means of days to flowering, racemes formed at first and second flowering slightly more than Parent one but closer to parent 2 in all crosses demonstrated the presence of midparent heterosis. Although, runner bean is self-pollinated there is a high degree of outcrossing (90\%). Therefore, the observed heterosis in parents can be exploited to develop high yielding runner bean varieties. From the results, the male parent Nyeri was selected as the best parent that flowers easily and abundantly hence giving high pod yield. This Parent can be utilized in future breeding programs of runner bean improvement. Among the parents, the dwarfs' accessions can also be used to develop early flowering and bush type runner beans.

\section{Genotype by environment influence on days to flowering}

The Ol Joro Orok region is predominantly cooler than Kabete and hence significant differences observed in days to flowering among populations at two locations was associated with temperature differences. The day length (12hrs) was constant across sites during flowering induction. Wallace et al., (1991) found that temperature alters the rate of vegetative development and higher temperatures cause nodes to flower in few days. They also concluded that genotypes with insensitivity to photoperiod tend to express a relatively small photoperiod-gene-causing delay to flowering. This was the case in short day parents and respective backcrosses, which delayed flowering in Ol Joro Orok with fewer days compared to White Emergo that took more than 10 days to flower at both sites. Thus there's high likelihood that short day parents and associated backcrosses are photoperiod insensitive to short day length and flowering may be delayed by few days when exposed to cooler climates. This also shows that short day photoperiod genes are easily fixable in improvement of runner bean for short day adaptation and developed varieties can be utilized in tropical climates.

\section{Genetic components}

Information on genetic inheritance of studied traits in runner bean was scanty. However, the results of this study were associated with reported findings of other crops. The results indicated that the mean effect $(\mathbf{m})$ of each cross was significant for all characters which reveal the difference in the inheritance of these traits among the local landraces vs the imported variety. The results also showed that the evaluated traits were quantitatively inherited since the segregating $F_{2}$ populations could not be grouped into classical ratios. 
Table 1. Mean performance of six populations in seven crossing combinations evaluated for days to flowering, number of racemes and pods at Kabete and OI Joro-Orok.

\begin{tabular}{|c|c|c|c|c|c|c|c|c|c|c|c|c|c|c|}
\hline \multirow{3}{*}{ Populations } & \multicolumn{14}{|c|}{ Days to $50 \%$ flowering } \\
\hline & \multicolumn{2}{|c|}{$\mathrm{W} \times \operatorname{Kin} 1$} & \multicolumn{2}{|c|}{$W \times \operatorname{Kin} 2$} & \multicolumn{2}{|c|}{$W \times \operatorname{Kin} 3$} & \multicolumn{2}{|c|}{ W x Nyeri } & \multicolumn{2}{|c|}{ W x Dwarf 1} & \multicolumn{2}{|c|}{ W x Dwarf 2} & \multicolumn{2}{|c|}{ W x Dwarf 3} \\
\hline & $\mathrm{KAB}$ & OJ & $\mathrm{KAB}$ & OJ & KAB & OJ & KAB & OJ & $\mathrm{KAB}$ & OJ & $\mathrm{KAB}$ & OJ & $\mathrm{KAB}$ & OJ \\
\hline$P_{1(\text { W. Emergo) }}$ & 53.7 & 55.7 & 54.5 & 53.9 & 54.8 & 56.5 & 55.0 & 57.7 & 54.4 & 54.5 & 53.8 & 53.5 & 53.6 & 56.8 \\
\hline $\mathrm{P}_{2}$ & 43.6 & 46.7 & 43.5 & 47.5 & 42.4 & 46.5 & 41.4 & 47.5 & 37.7 & 46.5 & 41.0 & 46.3 & 40.0 & 45.7 \\
\hline $\mathrm{F}_{1}$ & 47.0 & 48.8 & 47.0 & 50.0 & 44.9 & 49.6 & 45.0 & 48.3 & 47.5 & 50.5 & 43.5 & 48.3 & 43.9 & 49.6 \\
\hline $\mathrm{F}_{2}$ & 48.3 & 50.0 & 48.1 & 51.1 & 47.1 & 49.4 & 46.3 & 51.2 & 47.0 & 50.7 & 46.4 & 50.6 & 46.5 & 51.9 \\
\hline $\mathrm{BC}_{1} \mathrm{P}_{1}$ & 52.5 & 53.8 & 54.4 & 53.6 & 54.0 & 55.0 & 51.5 & 53.9 & 53.0 & 53.6 & 51.5 & 53.4 & 50.8 & 54.0 \\
\hline $\mathrm{BC}_{1} \mathrm{P}_{2}$ & 45.8 & 46.5 & 47.4 & 47.0 & 45.4 & 48.3 & 44.9 & 47.2 & 46.4 & 47.6 & 43.9 & 47.0 & 45.6 & 49.1 \\
\hline Mean & 48.5 & 50.1 & 49.4 & 50.2 & 51.0 & 50.9 & 47.4 & 50.9 & 46.3 & 50.5 & 46.3 & 49.6 & 46.6 & 50.9 \\
\hline CV (\%) & 7.1 & 3.8 & 7.5 & 3.0 & 7.5 & 7.3 & 6.0 & 5.4 & 6.1 & 5.5 & 6.7 & 5.2 & 5.6 & 5.1 \\
\hline \multirow[t]{3}{*}{$\mathrm{LSD}_{0.05}$} & 4.2 & 6.5 & 4.7 & 5.5 & 4.5 & 4.3 & 2.7 & 2.3 & 3.2 & 2.8 & 3.4 & 2.9 & 3.0 & 2.9 \\
\hline & \multicolumn{14}{|c|}{ Number of racemes at first flowering } \\
\hline & \multicolumn{2}{|c|}{ W $x$ Kin 1} & \multicolumn{2}{|c|}{$\mathrm{W} \times \operatorname{Kin} 2$} & \multicolumn{2}{|c|}{ W $x$ Kin 3} & \multicolumn{2}{|c|}{ W x Nyeri } & \multicolumn{2}{|c|}{ W x dwarf 1} & \multicolumn{2}{|c|}{ W x dwarf 2} & \multicolumn{2}{|c|}{ W x dwarf 3} \\
\hline Populations & $\mathrm{KAB}$ & OJ & $\mathrm{KAB}$ & OJ & $\mathrm{KAB}$ & OJ & KAB & OJ & KAB & OJ & $\mathrm{KAB}$ & OJ & $\mathrm{KAB}$ & OJ \\
\hline$P_{1 \text { (W.Emergo) }}$ & 3.1 & 4.1 & 3.3 & 3.0 & 3.8 & 6.7 & 2.7 & 1.0 & 4.0 & 3.2 & 2.5 & 1.9 & 2.7 & 2.9 \\
\hline $\mathrm{P}_{2}$ & 13.5 & 9.6 & 10.3 & 10 & 9.0 & 13.5 & 17.4 & 14.4 & 9.6 & 11.5 & 9.1 & 8.9 & 8.2 & 9.4 \\
\hline $\mathrm{F}_{1}$ & 7.3 & 9.3 & 9.9 & 7.3 & 9.6 & 8.4 & 12.1 & 7.7 & 5.6 & 8.6 & 9.3 & 8.9 & 7.6 & 8.0 \\
\hline$F_{2}$ & 9.7 & 10.3 & 8.4 & 9.0 & 8.9 & 11.5 & 9.8 & 8.5 & 8.4 & 10.2 & 6.4 & 9.4 & 7.4 & 9.1 \\
\hline $\mathrm{BC}_{1} \mathrm{P}_{1}$ & 4.8 & 8.0 & 6.1 & 6.6 & 8.2 & 8.5 & 8.1 & 7.0 & 5.5 & 8.6 & 6.8 & 7.3 & 6.8 & 4.5 \\
\hline $\mathrm{BC}_{1} \mathrm{P}_{2}$ & 9.8 & 12.8 & 11.2 & 9.5 & 10.8 & 9.0 & 13.6 & 12 & 8.2 & 12.1 & 8.6 & 9.6 & 9.0 & 9.1 \\
\hline Mean & 7.9 & 8.7 & 7.8 & 7.7 & 7.8 & 9.5 & 10.2 & 8.57 & 7.3 & 8.7 & 6.9 & 7.39 & 6.6 & 7.5 \\
\hline CV (\%) & 38.2 & 52.7 & 52.9 & 48 & 64.7 & 51.7 & 40.7 & 41.9 & 46.9 & 41.5 & 51.5 & 46.3 & 48.4 & 45 \\
\hline $\mathrm{LSD}_{0.05}$ & 3.6 & 5.4 & 4.3 & 4.1 & 5.4 & 5.7 & 3.5 & 2.9 & 3.9 & 3.6 & 3.9 & 3.7 & 3.7 & 3.8 \\
\hline & & & & & & Vumb & of race & hes at s & cond $\mathrm{f}$ & ering & & & & \\
\hline & $\mathrm{W} \times \mathrm{K}$ & & $\mathrm{W} \times \mathrm{K}$ & & $\mathrm{W} \times \mathrm{K}$ & & $W \times N$ & & $W \times c$ & If 1 & $W \times c$ & rf 2 & $W \times c$ & arf 3 \\
\hline Populations & $\mathrm{KAB}$ & OJ & $\mathrm{KAB}$ & OJ & $\mathrm{KAB}$ & OJ & $\mathrm{KAB}$ & OJ & $\mathrm{KAB}$ & OJ & $\mathrm{KAB}$ & OJ & $\mathrm{KAB}$ & OJ \\
\hline $\mathrm{P}_{1 \text { (W. Emergo) }}$ & 4.1 & 3.1 & 2.6 & 2.6 & 6.6 & 3.4 & 3.1 & 2.9 & 2.6 & 2.1 & 3.9 & 1.9 & 4.0 & 3.1 \\
\hline $\mathrm{P}_{2}$ & 13.1 & 11.1 & 12.3 & 11.9 & 16.1 & 16.1 & 18 & 13.8 & 2.5 & 9.5 & 7.4 & 6.0 & 6.7 & 5.3 \\
\hline $\mathrm{F}_{1}$ & 12.6 & 8.4 & 8.6 & 7.2 & 10.8 & 8.9 & 12.2 & 8.8 & 5.5 & 5.3 & 12.5 & 7.1 & 6.9 & 4.7 \\
\hline $\mathrm{F}_{2}$ & 14.1 & 8.7 & 10 & 8.6 & 10.5 & 12.9 & 9.5 & 10.6 & 13.2 & 8.0 & 10.0 & 9.1 & 9.5 & 7.6 \\
\hline $\mathrm{BC}_{1} \mathrm{P}_{1}$ & 5.3 & 5.3 & 7.0 & 6.6 & 7.3 & 7.3 & 5.8 & 7.9 & 7.0 & 6.2 & 7.0 & 6.5 & 6.8 & 5.5 \\
\hline $\mathrm{BC}_{1} \mathrm{P}_{2}$ & 10.3 & 10 & 10.4 & 14.3 & 10.6 & 9.7 & 13.6 & 14.3 & 6.4 & 8.7 & 8.0 & 10.7 & 11.2 & 8.6 \\
\hline Mean & 10.2 & 7.9 & 8.2 & 8.2 & 10.2 & 9.3 & 10.2 & 9.8 & 6.7 & 6.5 & 8.2 & 6.4 & 7.2 & 5.7 \\
\hline CV (\%) & 43.8 & 47.8 & 48.6 & 4.8 & 49.3 & 36.3 & 35.1 & 43.4 & 59.6 & 52 & 52.6 & 44.5 & 62.4 & 51 \\
\hline $\mathrm{LSD}_{0.05}$ & 5.4 & 4.5 & 4.1 & 3.8 & 5.9 & 3.9 & 3.2 & 1.8 & 4.6 & 3.4 & 4.7 & 3.1 & 5.2 & 3.2 \\
\hline & & & & & & & $\mathrm{Nu}$ & ber of $p$ & & & & & & \\
\hline & $\mathrm{W} \times \mathrm{K}$ & & $\mathrm{W} \times \mathrm{K}$ & & $W \times K$ & & $W \times N$ & & $W \times[$ & $\operatorname{arf} 1$ & $W \times[$ & irf 2 & $W \times[$ & $\operatorname{arf} 3$ \\
\hline Populations & $\mathrm{KAB}$ & OJ & $\mathrm{KAB}$ & OJ & $\mathrm{KAB}$ & OJ & $\mathrm{KAB}$ & OJ & $\mathrm{KAB}$ & OJ & $\mathrm{KAB}$ & OJ & $\mathrm{KAB}$ & OJ \\
\hline$P_{1 \text { (W. Emergo) }}$ & 7.1 & 9.0 & 9.9 & 8.3 & 9.6 & 8.8 & 8.5 & 11.5 & 10.2 & 9.2 & 6.6 & 5.4 & 7.3 & 7.6 \\
\hline$P_{2}$ & 21.6 & 26.7 & 18.8 & 28.2 & 22.3 & 38.9 & 38.1 & 33.2 & 26.7 & 30.5 & 24.3 & 28.2 & 32.4 & 24.7 \\
\hline $\mathrm{F}_{1}$ & 28.7 & 23.7 & 23.8 & 31.5 & 31.1 & 32.5 & 27.5 & 29.8 & 30.1 & 23.7 & 27.0 & 27.6 & 26.6 & 20.1 \\
\hline $\mathrm{F}_{2}$ & 21.9 & 24.4 & 20.4 & 29.9 & 26.6 & 30.9 & 28.9 & 26.7 & 25.5 & 24.2 & 24.9 & 22.9 & 24.9 & 24.9 \\
\hline $\mathrm{BC}_{1} \mathrm{P}_{1}$ & 9.0 & 13.0 & 15.9 & 10.4 & 10.3 & 11.8 & 10.9 & 12.1 & 21.5 & 21.8 & 14.0 & 12.0 & 12.8 & 15.0 \\
\hline $\mathrm{BC}_{1} \mathrm{P}_{2}$ & 26.0 & 23.0 & 20.8 & 28.3 & 13.4 & 21.8 & 30.1 & 23.4 & 23.8 & 29.0 & 23.0 & 27.7 & 23.2 & 23.1 \\
\hline Mean & 18.7 & 20.6 & 18.1 & 24.3 & 19.9 & 24.9 & 24.4 & 24.2 & 23.5 & 22.2 & 20.9 & 20.3 & 21.3 & 19.6 \\
\hline CV (\%) & 34.8 & 22.2 & 43.2 & 32.8 & 36.2 & 22.1 & 27.6 & 27 & 27.5 & 41.1 & 24.7 & 29.8 & 32.6 & 32.4 \\
\hline $\mathrm{LSD}_{0.05}$ & 7.9 & 5.4 & 7.6 & 8.9 & 8.4 & 6.4 & 5.9 & 5.4 & 7.4 & 9.2 & 5.6 & 6.6 & 7.9 & 7.2 \\
\hline
\end{tabular}

$\mathrm{P}_{1}=$ female parent (White Emergo), W= White Emergo $\mathrm{P}_{2}=$ male parents (Kin 1, Kin 2, Kin 3, Nyeri, Dwarf 1, Dwarf 2 and Dwarf 3), $\mathrm{BC}_{1} \mathrm{P}_{1}=$ backcross to female parent, $\mathrm{BC}_{1} \mathrm{P}_{2}=$ backcross to male parent, $K A B=$ Kabete, $O J=$ OlJoro-Orok, $L S D=$ least significance diference at $5 \%$ and $C V=$ coefficient of variation.
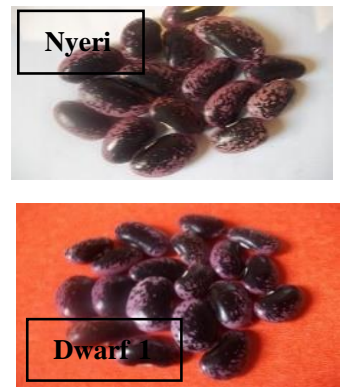
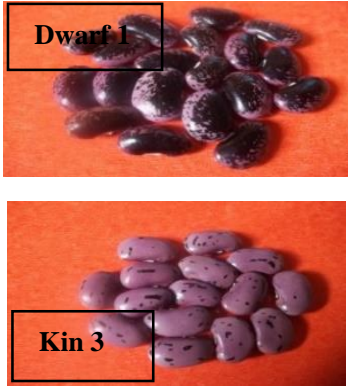
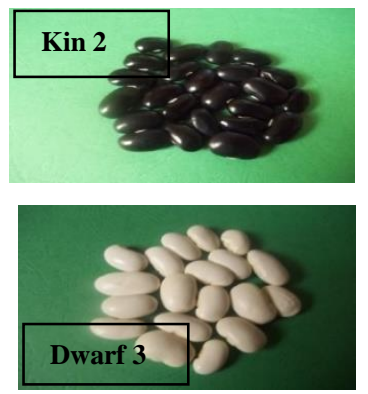

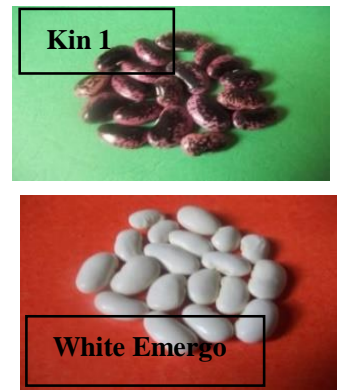

Fig 1. Seed colour of Parental lines used in the crossing. 
Table 2. Mean sum of squares for combined ANOVA for days to flowering at two locations.

\begin{tabular}{|c|c|c|c|c|c|c|c|c|}
\hline & & W $x$ Kin 1 & W $x$ Kin 2 & $W \times \operatorname{Kin} 3$ & W x Nyeri & W x Dwarf 1 & W x Dwarf 2 & W $x$ Dwarf 3 \\
\hline Source of & $\mathrm{df}$ & \multicolumn{7}{|c|}{ Mean sum of squares of Days to $50 \%$ flowering } \\
\hline Genotype & 5 & $39.49 *$ & 181.09* & $77.62 *$ & $512.83^{*}$ & $424.84^{*}$ & $42.84^{*}$ & $77.75^{*}$ \\
\hline Location & 1 & $39.02 *$ & $96.40 *$ & $69.82 *$ & 597.03* & 445.41* & $51.40 *$ & 132.92* \\
\hline $\mathrm{G} \times \mathrm{E}$ & 5 & $3.47 \mathrm{~ns}$ & $14.26 *$ & $0.79 \mathrm{~ns}$ & $9.87 \mathrm{~ns}$ & $65.43 *$ & $3.61^{*}$ & $1.49 \mathrm{~ns}$ \\
\hline
\end{tabular}

Table 3. Estimates of gene effects based on 3 parameter model for four traits in seven crosses of runner bean evaluated at two locations.

\begin{tabular}{|c|c|c|c|c|c|c|c|c|c|}
\hline \multicolumn{10}{|c|}{ Estimates of gene effects when fitted on 3-parameter Model $(m+a+d)$} \\
\hline \multirow[b]{2}{*}{ Cross } & \multirow[b]{2}{*}{ Model } & \multicolumn{2}{|c|}{ Days to $50 \%$ Flowering } & \multicolumn{2}{|c|}{ 1st flush Racemes } & \multicolumn{2}{|c|}{ 2nd Flush Racemes } & \multicolumn{2}{|c|}{ Number of pods } \\
\hline & & KAB & OJ & KAB & OJ & $\mathrm{KAB}$ & OJ & KAB & OJ \\
\hline \multirow{4}{*}{$\begin{array}{l}\frac{5}{x} \\
3\end{array}-1$} & $\mathrm{~m}$ & $46.7 \pm 0.8^{*}$ & $51.1 \pm 0.6 *$ & $8.4 \pm 0.6^{*}$ & $7.0 \pm 1.2 *$ & $8.3 \pm 1.6^{*}$ & $7.3 \pm 0.9 *$ & $13.3 \pm 2.5 *$ & $17.9 \pm 1.7 *$ \\
\hline & a & $3.1 \pm 1.8$ & $4.9 \pm 0.6^{*}$ & $-5.2 \pm 0.6^{*}$ & $-1.9 \pm 1.7$ & $-4.7 \pm 1.6 *$ & $-3.9 \pm 0.9 *$ & $-9.2 \pm 2.4 *$ & $-9.1 \pm 1.6^{*}$ \\
\hline & $\mathrm{d}$ & $2.0 \pm 3.4^{*}$ & $2.2 \pm 1.1$ & $-0.9 \pm 1.2$ & $2.6 \pm 3.1$ & $3.8 \pm 3.0$ & $1.3 \pm 1.8$ & $13.9 \pm 4.5^{*}$ & $5.13 \pm 3.1$ \\
\hline & $R^{2}$ & 0.79 & 0.88 & 0.86 & 0.66 & 0.88 & 0.68 & 0.66 & 0.74 \\
\hline \multirow{4}{*}{$\begin{array}{l}N \\
\stackrel{5}{x} \\
x \\
3\end{array}$} & $\mathrm{~m}$ & $49.7 \pm 0.8^{*}$ & $50.5 \pm 0.7 *$ & $4.4 \pm 15.3$ & $8.2 \pm 0.9 *$ & $7.8 \pm 0.7^{*}$ & $8.3 \pm 1.2 *$ & $14.5 \pm 1.2 *$ & $17.7 \pm 2.7 *$ \\
\hline & a & $5.8 \pm 0.8^{*}$ & $3.8 \pm 0.7^{*}$ & $-10.3 \pm 15.1$ & $-6.3 \pm 0.7^{*}$ & $-4.5 \pm 0.7^{*}$ & $4.9 \pm 1.2^{*}$ & $-4.6 \pm 1.2^{*}$ & $-11.6 \pm 2.6^{*}$ \\
\hline & $d$ & $-1.3 \pm 1.5^{*}$ & $-0.5 \pm 1.4^{*}$ & $53.5 \pm 28.4$ & $0.7 \pm 1.3$ & $1.7 \pm 1.2$ & $0.9 \pm 2.3$ & $9.5 \pm 2.2^{*}$ & $12.5 \pm 4.9 *$ \\
\hline & $\mathrm{R}^{2}$ & 0.83 & 0.7 & 0.89 & 0.87 & 0.81 & 0.57 & 0.74 & 0.69 \\
\hline \multirow{4}{*}{$\begin{array}{l}\stackrel{n}{\leftrightarrows} \\
\stackrel{5}{x} \\
3\end{array}$} & $\mathrm{~m}$ & $51.6 \pm 0.9 *$ & $51.6 \pm 1.0 *$ & NS & NS & $10.7 \pm 0.7 *$ & $9.9 \pm 1.4^{*}$ & $13.6 \pm 3.3 *$ & $21.9 \pm 3.3 *$ \\
\hline & $\mathrm{a}$ & $5.4 \pm 0.9 *$ & $5.4 \pm 0.9 *$ & NS & NS & $-4.5 \pm 0.7^{*}$ & $-5.6 \pm 1.4^{*}$ & $-5.6 \pm 3.3$ & $-14.6 \pm 3.2^{*}$ \\
\hline & $\mathrm{d}$ & $-1.9 \pm 1.7$ & $-2.0 \pm 1.8$ & NS & NS & $-1.1 \pm 1.4$ & $-0.5 \pm 2.3$ & $12.9 \pm 6.2^{*}$ & $5.9 \pm 6.1$ \\
\hline & $\mathrm{R}^{2}$ & 0.75 & 0.75 & NS & NS & 0.77 & 0.58 & 0.63 & 0.64 \\
\hline \multirow{4}{*}{$\begin{array}{r}\frac{\pi}{4} \\
\times \quad 3 \\
3\end{array}$} & $\mathrm{~m}$ & $46.9 \pm 1.1 *$ & $50.5 \pm 0.4 *$ & $46.9 \pm 1.1 *$ & $50.5 \pm 0.4 *$ & $4.9 \pm 1.7^{*}$ & $7.4 \pm 0.8^{*}$ & $18.4 \pm 1.2^{*}$ & $20.5 \pm 2.5^{*}$ \\
\hline & $\mathrm{a}$ & $7.9 \pm 1.2^{*}$ & $4.3 \pm 0.4^{*}$ & $7.9 \pm 1.2^{*}$ & $4.3 \pm 0.4^{*}$ & $-0.5 \pm 1.7$ & $-2.6 \pm 0.8^{*}$ & $-6.8 \pm 1.2^{*}$ & $-9.6 \pm 2.5^{*}$ \\
\hline & $d$ & $1.9 \pm 1.9 *$ & $0.1 \pm 0.8$ & $1.9 \pm 1.9 *$ & $0.1 \pm 0.8$ & $4.1 \pm 3.2$ & $-1.0 \pm 1.5$ & $10.9 \pm 2.2^{*}$ & $4.4 \pm 4.6$ \\
\hline & $R^{2}$ & 0.83 & 0.9 & 0.83 & 0.9 & 0.63 & 0.46 & 0.84 & 0.56 \\
\hline \multirow{4}{*}{ 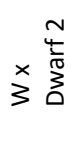 } & $\mathrm{m}$ & $48.0 \pm 0.7 *$ & $50.2 \pm 0.7 *$ & $5.9 \pm 0.6^{*}$ & $5.9 \pm 0.8^{*}$ & $5.3 \pm 0.8^{*}$ & $5.1 \pm 1.0 *$ & $15.8 \pm 1.4^{*}$ & $16.2 \pm 1.1 *$ \\
\hline & $\mathrm{a}$ & $6.7 \pm 0.7^{*}$ & $4.2 \pm 0.7^{*}$ & $-3.2 \pm 0.6^{*}$ & $-3.3 \pm 0.8^{*}$ & $-1.8 \pm 0.8^{*}$ & $-2.5 \pm 0.9 *$ & $-9.0 \pm 1.3^{*}$ & $-12.2 \pm 1.1^{*}$ \\
\hline & $\mathrm{d}$ & $-3.3 \pm 1.4^{*}$ & $-0.9 \pm 1.3^{*}$ & $3.1 \pm 1.2^{*}$ & $4.1 \pm 1.5^{*}$ & $6.5 \pm 1.6^{*}$ & $4.4 \pm 1.9 *$ & $11.4 \pm 2.5^{*}$ & $10.7 \pm 2.0 *$ \\
\hline & $\mathrm{R}^{2}$ & 0.89 & 0.77 & 0.73 & 0.67 & 0.65 & 0.47 & 0.86 & 0.93 \\
\hline \multirow{5}{*}{$\begin{array}{r}\frac{m}{4} \\
\times \\
3 \\
3\end{array}$} & $\mathrm{~m}$ & $47.4 \pm 0.5 *$ & $51.5 \pm 0.5 *$ & $5.9 \pm 0.6^{*}$ & $6.5 \pm 0.9 *$ & $6.5 \pm 1.2^{*}$ & $5.2 \pm 1.0^{*}$ & $18.8 \pm 1.6^{*}$ & $17.2 \pm 1.6$ * \\
\hline & $\mathrm{a}$ & $6.8 \pm 0.5^{*}$ & $5.5 \pm 0.5^{*}$ & $-2.6 \pm 0.6^{*}$ & $-3.3 \pm 0.9 *$ & $-2.1 \pm 1.2$ & $-1.5 \pm 1.0$ & $-12.2 \pm 1.5^{*}$ & $-8.5 \pm 1.6^{*}$ \\
\hline & $d$ & $-2.6 \pm 1.0$ & $-0.9 \pm 1.0$ & $2.8 \pm 1.0^{*}$ & $2.3 \pm 1.7$ & $2.6 \pm 2.2$ & $1.7 \pm 1.9$ & $5.6 \pm 2.96$ & $4.9 \pm 2.9$ \\
\hline & $R^{2}$ & 0.94 & 0.9 & 0.71 & 0.59 & 0.48 & 0.51 & 0.85 & 0.72 \\
\hline & $\mathrm{m}$ & $48.4 \pm 0.8 *$ & $52.5 \pm 0.5 *$ & $4.4 \pm 15.3$ & $8.2 \pm 0.9 *$ & $9.9 \pm 0.6^{*}$ & $9.2 \pm 0.9^{*}$ & $22.2 \pm 2.1^{*}$ & $20.3 \pm 2.2 *$ \\
\hline \multirow{3}{*}{$\sum_{3}^{x} \sum_{z}^{\frac{2}{2}}$} & $a$ & $6.3 \pm 0.8^{*}$ & $5.4 \pm 0.5^{*}$ & $-10.3 \pm 15.1$ & $-6.3 \pm 0.7^{*}$ & $-7.5 \pm 0.6^{*}$ & $-5.6 \pm 0.9 *$ & $-15.3 \pm 2.1^{*}$ & $-11 \pm 2.2^{*}$ \\
\hline & $d$ & $-3.2 \pm 1.6$ & $-3.5 \pm 1.0$ & $53.5 \pm 28.4$ & $0.7 \pm 1.3$ & $0.9 \pm 1.1$ & $1.4 \pm 1.7$ & $3.9 \pm 3.9$ & $5.9 \pm 3.9$ \\
\hline & $\mathrm{R}^{2}$ & 0.85 & 0.91 & 0.89 & 0.87 & 0.94 & 0.77 & 0.83 & 0.71 \\
\hline
\end{tabular}

$\mathrm{W}=$ White Emergo, $\mathrm{R}^{2}=$ coefficient of determination, $\mathrm{KAB}=$ Kabete, $\mathrm{OJ}=\mathrm{Ol}$ Joro-orok, $\mathrm{m}=$ mean effect $\mathrm{a}=$ additive effect and $\mathrm{d}=$ dominance effect and $\mathrm{NS}=\mathrm{Means}$ the populations did not show significant differences for this cross at $(P \leq 0.05)$ hence excluded from this analysis

Table 4. Heterosis and heritability estimates of traits studied.

\begin{tabular}{|c|c|c|c|c|c|c|c|c|c|}
\hline \multirow[t]{2}{*}{ Cross } & \multirow[b]{2}{*}{ Location } & \multicolumn{2}{|c|}{ Days to $50 \%$ flowering } & \multicolumn{2}{|c|}{ 1st flowering Racemes } & \multicolumn{2}{|c|}{ 2nd flowering racemes } & \multicolumn{2}{|c|}{ Number of pods } \\
\hline & & HBS (\%) & $\mathrm{BPH}(\%)$ & HBS (\%) & $\mathrm{BPH}(\%)$ & HBS (\%) & $\mathrm{BPH}(\%)$ & HBS (\%) & $\mathrm{BPH}(\%)$ \\
\hline \multirow[t]{2}{*}{ W x Kin1 } & Kab & 81.70 & -41.5 & 73.65 & -30.4 & 72.63 & -20.9 & 64.20 & 99.7 \\
\hline & OJ & 78.39 & -13.8 & 82.09 & -39.9 & 78.58 & 57.3 & 77.33 & 32.7 \\
\hline \multirow[t]{2}{*}{ W $x$ Kin2 } & Kab & 78.56 & -3.2 & 73.08 & 24.1 & 82.67 & 62.7 & 76.40 & 65.3 \\
\hline & OJ & 85.77 & 20.3 & 83.42 & -6 & 86.22 & 5.7 & 74.55 & 72.9 \\
\hline \multirow[t]{2}{*}{ W $x$ Kin3 } & Kab & 77.22 & 93.4 & NS & NS & 75.89 & 12.6 & 74.60 & 95.5 \\
\hline & OJ & 66.63 & 56.9 & NS & NS & 78.99 & -6.5 & 73.95 & 36.2 \\
\hline \multirow[t]{2}{*}{ W xNyeri } & Kab & 78.05 & -36.1 & 78.36 & -15.7 & 80.87 & 88.3 & 78.07 & 18.1 \\
\hline & OJ & 91.02 & 29.9 & 83.54 & -44.5 & 93.21 & -67 & 77.16 & 33.6 \\
\hline \multirow[t]{2}{*}{ W x dwf1 } & Kab & 79.88 & -6.7 & 71.15 & -19.6 & 68.67 & 1.6 & 73.01 & 63.1 \\
\hline & OJ & 72.62 & 74.4 & 78.13 & 40.4 & 79.40 & 10.5 & 81.11 & 19.4 \\
\hline \multirow[t]{2}{*}{ W x dwarf 2} & Kab & 77.39 & -39.7 & 81.72 & -36.1 & 79.91 & -2.9 & 68.10 & 75.3 \\
\hline & OJ & 78.95 & 12.9 & 73.71 & 24.1 & 68.69 & 33.9 & 77.67 & 64.3 \\
\hline \multirow[t]{2}{*}{ W x dwarf 3} & Kab & 80.38 & 99.6 & 89.54 & -35.6 & 73.39 & -12.1 & 72.18 & 34.3 \\
\hline & OJ & 69.96 & -31.8 & 71.44 & -18.4 & 75.76 & 53.0 & 76.58 & 24.2 \\
\hline
\end{tabular}


Table 5. Climatic conditions during the assumed flower induction period at Kabete and Ol Joro-Orok.

\begin{tabular}{llllll}
\hline Site Induction period & Altitude & \multicolumn{2}{l}{ Mean air temp. $\left({ }^{\circ} \mathrm{C}\right)$} & \multicolumn{2}{l}{ Photoperiod(hr) } \\
\hline & & 2013 & 2014 & 2013 & 2014 \\
\cline { 3 - 6 } Ol Joro orok (April-May) & $2300 \mathrm{~mm}$ & $14.5(13-16)$ & $15(15-15.2)$ & 12.0 & 12.0 \\
Kabete (April-May) & $1820 \mathrm{~mm}$ & $18.6(18-19)$ & $18(17-19)$ & 12.0 & 12.0 \\
\hline
\end{tabular}

Table 6. Coefficients of $\alpha_{k}$ and $\delta_{k}$ utilized for the construction of different models in generation mean analysis based on Mather and Jinks, 1971.

\begin{tabular}{lllllll}
\hline & \multicolumn{7}{c}{ Genetic effects } \\
\hline Generation & $\mathrm{m}$ & $\mathrm{a}$ & $\mathrm{d}$ & $\mathrm{aa}$ & $\mathrm{ad}$ & $\mathrm{dd}$ \\
\hline $\mathrm{P}_{1}$ & 1 & -1 & 0 & 1 & -1 & 0.25 \\
$\mathrm{P}_{2}$ & 1 & 1 & 0 & 1 & 1 & 0.25 \\
$\mathrm{~F}_{1}$ & 1 & 0 & 1 & 0 & 0 & 0.25 \\
$\mathrm{~F}_{2}$ & 1 & 0 & 0.5 & 0 & 0 & 0 \\
$\mathrm{BC}_{1} \mathrm{P}_{1}$ & 1 & -0.5 & 0.5 & 0.25 & 0 & 0 \\
$\mathrm{BC}_{1} \mathrm{P}_{2}$ & 1 & 0.5 & 0.5 & 0.25 & 0 & 0 \\
\hline
\end{tabular}

Where, $\mathbf{m}=$ mean effect of parental homozygotes, a=additive effects, $\mathbf{d}=$ dominance effect, aa= additive $\mathrm{x}$ additive effects, ad=additive $\mathrm{x}$ dominance effects and $\mathbf{d d}=$ dominance $\mathrm{x}$ dominance effects.

Additive-dominance model $(m+a+d)$ was found to adequately explain gene action controlling days to $50 \%$ flowering, the number of racemes and pods formed compared to the digenic interactions in all crosses. This shows that epistatic effects were not important in the inheritance of traits studied in the crosses evaluated as indicated by the joint scaling test. In addition, the dominance parameter [d] was not significant for all evaluated traits in all crosses except in White Emergo x Kin 1 and White Emergo $\times$ Dwarf 2 in the number of racemes and number of pods indicating about $90 \%$ influence of additive effects in these traits. The joint scaling test and t significance tests indicated the adequacy of the model $(m+a+d)$. However, $R^{2}$ values not fitting exactly to $99 \%$ or $100 \%$ was attributed to experimental error as suggested by Ceballos et al., (1998) that experimental error lowers the percentage of the model to fit well. The prevalence of additive or additivedominant models other than epistatic effects has also been found by Rainey and Griffiths, (2005) in generation means analysis conducted for such traits in common bean.

When estimating each gene effect, the additive effects were found to majorly influence days to flower in all crosses as opposed to dominance or epistatic effects concurring with the results of Arunga et al., 2010; Silva et al., 2004 in their study on snap beans. Additive effects accounted for more influence in this study similar to the findings of Mendes et al., 2008 who found additive $x$ dominance effects to influence days to $50 \%$ flowering but further revealed that the dominance effects were less important in controlling the number of days to $50 \%$ flowering and their effect was to reduce the number of days to flowering. The positive dominance effects in the number of racemes at first and second flowering and number of pods indicated the existence of partial dominance in the latter traits in all crosses. The results revealed that numbers of racemes formed at both flowering stages were significantly influenced by additive effects although the additive and dominance effects were important in the cross; White $x$ Dwarf 2. Das et al., 2014 also found that number of inflorescences per plant and numbers of buds per inflorescences in dolichos are predominantly influenced by additive genetic effects. The results of this study showing additive effects sgnificantly influencing the number of pods per plant were similar to findings of Arunga et al., 2010 in snap beans and Das et al., 2014 in dolichos. This indicates a strong evidence that short day photoperiod can be easily inherited when developing breeding schemes for the selection of superior runner bean lines as the studied traits are mainly influenced by the fixable gene effect. Consequently, selection of these traits will be useful to start at early segregating generations (Hinkosa et al., 2013).

\section{Heritability and heterosis of studied traits}

High broad sense heritability observed for all traits showed that rapid progress can be made when using selection procedures that are dependent on the phenotype (Acquaah, 2007). From these findings, it was noted that heritability of a number of days to flowering was high as revealed in the results however the magnitude of heritability in number of pods was highly influenced by genetic materials and the environment where they were evaluated. Presence of positive heterosis that was observed in the number of pods is a clear evidence of the manifestation of hybrid vigour hence parents that yield more pods could be utilized in improving yield in runner beans.

\section{Materials and Methods}

\section{Genetic materials used}

The breeding materials used were seven short day grain type local landraces namely; Nyeri, Kin 1, Kin 2, Kin 3, Dwarf 1, Dwarf 2, and Dwarf 3 which were crossed as male parents $\left(P_{2}\right)$ to a long day female parent $\left(P_{1}\right)$, White Emergo. The seven short day grain type parents were local landraces which flower easily under the 12 hour short day length in Kenya and hence were chosen as male parent to donate short-photoperiod genes to the preferred long day variety by exporters and consumers. These accessions were collected from farmers in Nyeri, Kinangop and OlJoro-Orok areas in Kenya and hence the designation of the names. White Emergo is a long day imported vegetable variety, flowering in $16 \mathrm{hrs}$ of day length, high yielding with straight, tender pods ranging from $(18-30 \mathrm{~cm})$ hence highly preferred by exporters and consumers of vegetable pods. However, it 
lacks the short-photoperiod genes and hence cannot flower in short-day conditions. The growth habit of all parents was climbing vines except for dwarf parents which had a bush growth type. White Emergo and Dwarf 3 had white flowers associated with the white colour of the standard while Kin 1, Kin 2, Kin 3, Nyeri, Dwarf 1 and Dwarf 2 had red flowers with a scarlet standard. All the local landraces collections flowers easily in Kenya under natural 12 hour day length, unlike White Emergo which delays flowering and need additional artificial light of 4 hours to trigger flowering. The seed colour of the parental lines varied from mono coloured to speckled seed patterns as shown in Fig 1.

\section{Trial sites and Population development in the screen house}

Population development was done in an insect proof screen house at Kabete Field Station, Kenya to minimize outcrossing. The screen house was adjacent to flood light (400watts) which provided additional 4hrs of light to facilitate flowering of White Emergo for population development. The flood lights were put on from $6 \mathrm{pm}$ to $6 a m$. Seven short-day male parents were crossed with one female parent (White Emergo) to generate seven crosses; (White Emergo $\times$ Nyeri), (White Emergo $\times$ Kin 1), (White Emergo $\mathrm{x}$ Kin 2), (White Emergo $\mathrm{x}$ Kin 3), (White Emergo $\mathrm{x}$ Dwarf 1), (White Emergo $\times$ Dwarf 2), and (White Emergo $\mathrm{x}$ Dwarf 3). The six generations were developed through a stepwise crossing from December 2012 to December 2013. $F_{1}$ were advanced to $F_{2}$ and part of it also back-crossed to long-day and short-day parents hence creating $\mathrm{BC}_{1} \mathrm{P}_{1}$ and $B_{1} P_{2}$. The eight parental lines were planted in pots at intervals of one week to ensure synchronization of flowering in the greenhouse and availability of adequate pollen during pollination. Pots were irrigated manually twice in the morning and evening using watering cans. Populations were evaluated in the field at Kabete $(1840 \mathrm{~m}$ a.s.l) University of Nairobi field station and KALRO- OlJoro-Orok (2300m a.s.l) under the 12 hour natural day length. No controlled day length was provided during field evaluation. The two locations receive a mean annual rainfall of about $1000 \mathrm{~mm}$. The average temperatures are $13.7-24.3^{\circ} \mathrm{C}$ at Kabete and $10^{\circ} \mathrm{C}-22^{\circ} \mathrm{C}$ at Ol Joro-Orok (Jaetzold et al., 2006). The mean temperature and day length during flower inductions presented are presented in Table 5.

\section{Field evaluation}

The six generations of each cross were planted as a separate experiment at Kabete and OlJoro-orok. The experiment was laid out in a modified complete randomized block design with two replications. Rows in a plot were $3 \mathrm{~m}$ long. Number of rows per plot varied with treatments. Backcrosses $\left(B C_{1} P_{1}\right.$ and $\left.B C_{1} P_{2}\right)$ were planted in plots with two rows. $F_{1}$, female parent $\left(P_{1}\right)$, male parent $2\left(P_{2}\right)$ were on a 3-row plot, while $F_{2}$ populations were planted in the 4-row plot. The intra-row spacing was $20 \mathrm{~cm}$ and Inter-row spacing was $50 \mathrm{~cm}$. The number of plants evaluated varied depending on the treatment whereby $\mathrm{BC}_{1} \mathrm{P}_{1}$ and $\mathrm{BC}_{1} \mathrm{P}_{2}$ had 10 plants, $\mathrm{F}_{1}, \mathrm{P}_{1}$ and $P_{2}$ had 15 plants and $F_{2}$ had 20 plants per replication. Diammonium phosphate fertilizer (DAP) was used during planting at a rate of $60 \mathrm{~kg} \mathrm{ha-}{ }^{1}$. Because runner bean is a climbing bean, a string was used to support the plant whereby each individual plant was tied with a string (at the base of the plant) to a top placed heavy weight wire suspended horizontally across the row. The wire was supported by sturdy wooden poles on each side of the row. Insect pests were controlled when necessary in addition to manual weeding and supplementary irrigation. Data was collected on aspects related to flowering and yield this included duration to $50 \%$ flowering, number of flowers (counted in racemes) and number of pods per plant. Duration to $50 \%$ flowering was recorded as the number of days after planting to the date when $50 \%$ of plants had one or more open flowers. Runner bean sets flowers in two flushes and therefore number of flowers were counted on a single plant basis at $1^{\text {st }}$ flush and $2^{\text {nd }}$ flush of flowering at both sites. Since the runner bean inflorescence is grouped in a raceme, flower counting was based on a number of racemes with one raceme estimated to have 15-20 single flowers. Pods from each plant were counted when the plants had reached physiological maturity in 270 days at OlJoroOrok and 150 days ta Kabete. The numbers of pods were based on cumulative racemes formed in both flowering flushes.

\section{Statistical and genetic analysis}

Data for each cross and location was analyzed separately as described by (Checa et al., 2006). Analysis of variance (ANOVA) was conducted using Genstat statistical software14 ${ }^{\text {th }}$ edition (VSN international, 2011) where the $F$ test showed significant differences among generations, Tukey's test was used to separate the means at $(P \leq 0.05)$. Analysis of variance was performed to determine the significance of genotypic effect followed by genetic analyses. Generation means analysis was applied on traits that showed significance differences among generations to determine the mode of inheritance of short-day photoperiod. This analysis was accomplished in the following steps;

1. The traits that showed significant differences in the ANOVA analysis among the generations at both locations were subjected to generation mean analysis proposed by Mather and Jinks (1971). For each given cross, trait and at each location, generation means was expressed in terms of genetic effects using the equation below; $\mathbf{g}_{\mathrm{k}}=\mathbf{m}+\left(\alpha_{\mathrm{k}}\right) \mathbf{a}+$ $\left(\delta_{k}\right) \mathbf{d}+\left(\alpha_{k}\right) 2 a a+\left(\alpha_{k} \delta_{k}\right)$ ad $+\left(\delta_{k}\right) 2$ dd . Where $g_{k}=$ mean of generation $k, m=$ mean of the parental homozygotes, $\alpha_{k}$ and $\delta_{k}=$ coefficients determined by the degree of relationship of generation $\mathbf{k}, \mathbf{a}=$ additive gene effects, $\mathbf{d}=$ dominance gene effects, aa = epistatic effects of additive $\mathrm{x}$ additive type, $\mathbf{a d}=$ epistatic effects of additive $\mathrm{x}$ dominant type and $\mathbf{d d}=$ epistatic effects of dominant $x$ dominant type.

2. The coefficients shown in Table 6 , means and the variances of each of the six generations in each site were submitted to regression analysis. Linear regression analysis was carried out using the statistical package Genstat $14^{\text {th }}$ edition by weighting based on the inverse of the variance of means and the matrix of the coefficient of genetic effects (Mather and Jinks, 1971).

3. Both the $3(m+a+d)$ and $6(m+a+d+a a+a d+d d)$ parameter models were tested. Mather and Jinks (1982) scaling tests were employed to determine the adequacy of a 3-parameter model $(\mathrm{m}+\mathrm{a}+\mathrm{d})$ as described by Hinkosa et al., 2013 and Zdravkovic et al., 2011 where each scale was calculated as follows; 
$\mathrm{A}=2 \overline{\mathrm{BC}_{1} \mathrm{P}_{1}}-\overline{\mathrm{F}_{1}}-\overline{\mathrm{P}_{1}}$ and $\mathrm{V}_{\mathrm{A}}=4 \mathrm{~V}_{\mathrm{BC}_{1} \mathrm{P}_{1}}+\mathrm{V}_{\mathrm{P}_{1}}+\mathrm{V}_{\mathrm{F}_{1}}$ $\mathrm{B}=2 \overline{\mathrm{BC}_{1} \mathrm{P}_{2}}-\overline{\mathrm{F}_{1}}-\overline{\mathrm{P}_{2}}$ and $\mathrm{V}_{\mathrm{B}}=4 \mathrm{~V}_{\mathrm{BC}_{1} \mathrm{P}_{2}}+\mathrm{V}_{\mathrm{P}_{2}}+\mathrm{V}_{\mathrm{F}_{1}}$ $\mathrm{C}=4 \overline{\mathrm{F}_{2}}-2 \overline{\mathrm{F}_{1}}-\overline{\mathrm{P}_{1}}-\overline{\mathrm{P}_{2}}$ and $\mathrm{V}_{\mathrm{C}}=16 \mathrm{~V}_{\mathrm{F}_{2}}+4 \mathrm{~V}_{\mathrm{F}_{1}}+\mathrm{V}_{\mathrm{P}_{1}}+\mathrm{V}_{\mathrm{P}_{2}}$ Where; $\mathrm{V}_{\mathrm{P}_{1}}, \mathrm{~V}_{\mathrm{P}_{2}}, \mathrm{~V}_{\mathrm{F}_{1}}, \mathrm{~V}_{\mathrm{F}_{2}}, \mathrm{~V}_{\mathrm{BC}_{1} \mathrm{P}_{1}}$ and $\mathrm{V}_{\mathrm{BC}_{1} \mathrm{P}_{2}}$ were the variances estimated

The values of T- test were calculated as follows:

$$
\begin{gathered}
\pm \mathrm{t}=\frac{\text { Deviation }}{\text { standard error }}=\frac{\text { Deviation (Values of A or B or C) }}{\sqrt{\text { Variation of deviation }}} \\
\pm \mathrm{t}_{\mathrm{A}}=\frac{\mathrm{A}}{\sqrt{\mathrm{V}_{\mathrm{A}}}} \text { and } \mathrm{t}_{\mathrm{B}}=\frac{\mathrm{B}}{\sqrt{\mathrm{V}_{\mathrm{B}}}} \text { and } \mathrm{t}_{\mathrm{c}}=\frac{\mathrm{C}}{\sqrt{\mathrm{V}_{\mathrm{C}}}}
\end{gathered}
$$

In each scaling test, the degree of freedom was the sum of the degrees of freedom of various generations involved in each location and the t-test was done at 1 and $5 \%$ probability level to test significance of each scale. There was a significance difference if $t_{c a l}>t_{t a b}$ and no significance if $t_{c a l}<t_{t a b}$. If at least one value from A, B, C set were statistically significant based on t-test then the 3-parameter model $(m+a+d)$ was declared inadequate therefore indicating the presence of non- allelic or epistatic effect.

4. The significance of each genetic estimate (effect) either additive, dominance or epistatic effects in the chosen model were evaluated by utilizing the significance of the t-test at $5 \%$ significance level.

5. Heritability of the traits was also calculated as follows: Broad sense heritability:

$H_{B S}^{2}=\left(\sigma^{2} G_{(F 2)} / \sigma_{(F 2)}^{2}\right) * 100$, where: $\sigma^{2}{ }_{P 1}=$ variance of parent $1 ; \sigma_{P 2}^{2}=$ variance of parent $2 ; \sigma_{F 1}^{2}=$ variance of $F_{1} ; \sigma^{2} F_{2}=$ variance of $F_{2}$ generation; $\sigma^{2}{ }_{\mathrm{BC} 1 \mathrm{P} 1}=$ variance of backcross to parent 1 and $\sigma^{2} \mathrm{BC}_{1} \mathrm{P}_{2}=$ variance of backcross to parent

6. Better parent heterosis (BPH) was calculated as; $\mathrm{BPH}(\%)=$ $\left(\left(F_{1}-B P\right) / B P\right) * 100$, Where, $F_{1}=$ Mean value of the $F_{1}$ progeny and $\mathrm{BP}=$ Mean value of the better parent

\section{Conclusion}

This study was the first step in determining gene action and possible breeding implications of runner beans in Africa. White Emergo (long day parent) delayed to flower and had fewer racemes and pods revealing how flowering of this variety is constrained under short day conditions. Short day parents and associated backcrosses in this study are likely to be photoperiod insensitive to short day length and flowering may be delayed by few days when exposed to cooler climates. Results from this study showed the predominance of additive effect influence in control of days to flowering, number of racemes and number of pods in runner beans. This shows a strong evidence that short day photoperiod can be easily inherited when developing breeding schemes for selection of superior runner bean lines. It also implies that improvement of this crop will involve selection in early generation stages because additive effects would give a better response. We, therefore recommend selection procedures or modification of such methods including pedigree, single seed/pod descent and backcross and therefore breeders should use the methods to effectively select for associated traits to short day adaptation.

\section{Acknowledgement}

This research was supported by funds from Regional Universities Forum for Capacity Building in Agriculture (RUFORUM) and the authors are very grateful for the support. The University of Nairobi and KALRO are also acknowledged for offering experimental fields and support.

\section{References}

Acquaah G (2007) Principles of plant genetics and breeding $2^{\text {nd }}$ ed. Wiley-Blackwell publishing.

Arunga EE, Van-Rheenen HA, Owuoche OJ (2010) Diallel analysis of snap (Phaseolus Vulgaris L.) varieties for important traits. Afr J Agri Res. 5(15):1951-1957.

Bicer BT Sakar (2008) Heritability and gene effects for yield and yield components in chickpea. Hereditas. 145: 220224.

Brink M (2006) Cereals and pulses book. Plant Resources of Tropical Africa (PROTA). Accessed at

Caiger S (1995) Runner bean profile for Sri-Lanka. The agroenterprise development project, Colombo, Sri-Lanka. Miscellaneous reports.

Ceballos H, Pandey S, Narro L and Perez-Velazquez JC (1998) Additive-dominant and epistatic effects for maize grain yield in acid and non-acid soils. Theo Appl Genet. 96:662668.

Checa O, Ceballos H, Blair MW (2006) Generation means analysis of climbing ability in common bean (Phaseolus vulgaris L.). J Hered. 97:456-465.

Das I, Seth T, Durwas SV, Dutta S, Chattopadhyaya A, Singh B (2014) Gene action and combining ability for yield and yield components traits in dolichos bean (Dolichos lablab var.typicus). SABRAO J Breed Genet. 46: 293-304.

Ganesh SK, Sakila M (1999) Generation mean analysis in sesame (Sesamum Indicum L.) crosses. Sesame and Safflower Newsletter. 14:8-14.

Hinkossa A, Gebeyehu S, Zeleke H (2013) Generation mean analysis and heritability of drought resistance in common bean (Phaseolus vulgaris.L.). Afr J Agri Res. 8:1319-1329.

Jaetzold R, Schmidt H, Hornetz B, Shisanya B (2006) Farm management hand book of Kenya, Vol II. Nairobi, Kenya Ministry of Agriculture. Natural conditions and farm management information of Central Kenya.

Khodambashi M, Bitaraf N, Hoshmand S (2012) Generation mean analysis for grain yield and its related traits in lentil. J Agr Sci Tech. 14:609-616.

Kornegay J, White JW, Dominguez JR, Tejada G, Cajiao G (1993) Inheritance of photoperiod response in andean and mesoamerican common bean. Crop Sci. 33:977-984.

Lumpkin TA, McClary DC (1994) Breeding and genetics in adzuki bean: botany, production and uses. $C A B$ International, pp. 119-155.

Martin FW (1984) Handbook of tropical food crops pp. 4849.CRC press, Australia.

Mather K, Jinks L (1971) Biometrical genetics. Cornell University Press, Ithaca, New York, USA.

Mendes MP, Botelho FB, Ramalho MA, Breu AFBA, and Furtini IV (2008) Genetic control of the number of days to flowering in common bean. Crop Breed Appl Biot. 8: 279282.

Purseglove JW (1987) Tropical crops book (Dicotyledons). Cambridge University Press. Wiley, New york. 
Rainey KM, Griffiths PD (2005) Inheritance of heat tolerance during reproductive development in snap bean (Phaseolus vulgaris L.). J Amer Soc Hort Sci. 130:700-706

Silva PM, Junior AT, Rodrigues R, Pereira GM, Viana AP (2004) Genetic control and morpho-agronomic traits in snap bean. Arch Biol Technol. 47: 855-882.

VSN International (2011) Genstat for Windows $14^{\text {th }}$ Edition. VSN International, Hemel Hempstead, UK. Web page: Genstat.co.uk.

Thomas and Vince-Prue (1997) Photoperiodism in plants $2^{\text {nd }}$ edition, Academic Press, London.
Wallace DH, Gniffke PA, Masaya PN, Zobel RW (1991) J Amer Soc Hort Sci. 116(3):534-543.

Horticultural Crops Development Authority (2013) Horticulture Validated report. 2013.

Mather K, Jinks JL (1982) Introduction to Biometrical Genetics. Chapman and Hall Ltd., London.

Zdravković J, Pavlović N, Girek Z, Jokanović M , Savić D, Zdravković M , Cvikić D (2011) Generation mean analysis of yield components and yield in tomato (Lycopersicon esculentum MILL.) Pak J Bot. 43(3): 1575-1580. 